\title{
Gastrointestinal Complications after Allogeneic Hematopoietic Stem Cell Transplant: A Multidisciplinary Approach with Early Endoscopic Evaluation
}

\author{
Giuseppe Tarantino ${ }^{1, *}, \mathbb{D}$, Francesco Saraceni ${ }^{2}$, Giorgia Mancini ${ }^{2}$, Monica Poiani ${ }^{2, \dagger}$, Luca Maroni ${ }^{1, \mathbb{D}}$, \\ Gaia Goteri ${ }^{3}$ (D), Ilaria Scortechini ${ }^{2}$, Alessandro Fiorentini ${ }^{2}$, Maria Vittoria Dubbini ${ }^{2}$, Francesco Marini ${ }^{4}$, \\ Luigi Daretti ${ }^{4}$, Marco Marzioni ${ }^{1}$, Emanuele Bendia ${ }^{4}$, Antonio Benedetti ${ }^{1}$, Attilio Olivieri ${ }^{2}$ \\ ${ }^{1}$ Clinica di Gastroenterologia, Epatologia ed Endoscopia Digestiva d'Urgenza, Via Conca 71, Ospedali Riuniti Ancona, Italy \\ ${ }^{2}$ Clinica Ematologica, Dipartimento di Medicina Interna, Ospedali Riuniti Ancona, Italy \\ ${ }^{3}$ Anatomia Patologica, Dipartimento di Medicina Interna, Ospedali Riuniti Ancona, Italy \\ ${ }^{4}$ Malattie dell'Apparato Digerente, Endoscopia Digestiva e Malattie Infiammatorie Croniche Intestinali, Ospedali Riuniti Ancona, Italy
}

ARTICLE IN F O
Article History
Received 15 April 2021
Accepted 26 August 2021
Keywords
Gastro-intestinal complications
$\quad$ (GICs) in allogeneic
hematopoietic stem cell
transplant (allo-HSCT)
graft-versus-host-disease (GVHD)
mucositis
endoscopy
intestinal histology

\section{INTRODUCTION}

The clinical course of patients undergoing allogeneic hematopoietic stem cells transplant (allo-HSCT) is frequently complicated by several conditions affecting the gastro-intestinal (GI) tract. GI complications (GICs) represent the major cause of morbidity and mortality after allo-HSCT [1]. The global incidence of GICs is probably underestimated, because of their high variability and complexity. Further, when evaluating symptoms arising from the GI system, the differential diagnosis may be challenging, as different conditions such as graft-versus-host disease (GVHD), infections, drug toxicity and acute exacerbation of previous GI diseases may overlap. Diagnostic assessment is often a challenge, due to the complexity of clinical settings in which several conditions can

"Corresponding author.Email: giutar77@gmail.com

'SC Ematologia A.S.U. Ospedale Maggiore, Trieste, Italy

Peer review under responsibility of the International Academy for Clinical Hematology Data availability statement: The datasets generated during and/or analysed during the current study are available from the corresponding author [GT], on reasonable request. affect the same patient or produce non-specific signs and symptoms (i.e., diarrhea and abdominal pain) [2-4]. GVHD represents the main cause of non-relapse mortality (NRM) in patients undergoing allo-HSCT and requires a prompt diagnosis and therapeutic intervention. Nevertheless, different conditions other than GVHD affecting the GI system cause significant morbidity for patients undergoing allo-HSCT. The use of chemo-radiotherapy and prolonged antibiotic treatment may lead to mucosal damage in the GI apparatus, with loss of oral and intestinal microbiota diversity. The global incidence of mucositis in patients undergoing allo-HSCT ranges usually between $60 \%$ and $100 \%$. Total body irradiation (TBI), alkylating agents and methotrexate are particularly toxic for the GI mucosa. Mucositis is responsible for severe pain and extensive ulcers, and the damage of the protective mucosal barrier results in the dissemination of pathogens, leading to high risk of bacterial dissemination and sepsis [5-7]. The tissue damage consequent to the preparatory regimen, in association with severe and prolonged thrombocytopenia, may promote GI bleeding, which represents a rare but severe complication after allo-HSCT [8]; on 
the contrary, ischemic GI complications are less common but even more challenging [9].

Prolonged neutropenia and immunosuppressive drugs increase the risk of GI infections: it is estimated that up to $40 \%$ of patients undergoing allo-HSCT develops bacterial, viral or fungal GI infections [10].

In the present study, we have retrospectively evaluated GICs in 122 consecutive patients receiving allo-HSCT in the last 5 years, by using a clinical algorithm, based on a systematic interdisciplinary approach, supported by a dedicated endoscopic service. The secondary goal was to focus onto the differential diagnosis between GVHD and the other GI conditions, based on a standardized quick endoscopic/histological assessment. For this purpose, we have also calculated the diagnostic accuracy of this approach for the GI-GVHD.

\section{MATERIALS AND METHODS}

\subsection{Patients}

Since January 2015 an interdisciplinary approach for post-transplant GICs was implemented at our institution, with a dedicated GI endoscopic unit, to promptly diagnose GICs following allo-HCST. We included in the analysis patients who received allo-HSCT for different hematological diseases from January 2015 to August 2019.

During the pre-transplant phase, we have recorded and analyzed the following data:

- Age at diagnosis, gender, infective and immunity state related to hepatotropic viruses [HBV, HCV, HIV, Cytomegalovirus $(\mathrm{CMV})], \mathrm{AB} 0 / \mathrm{Rh}$ status of blood group.

- Pre-existing co-morbidities with particular interest in gastroenterological conditions, such as chronic hepatitis, diabetes and metabolic syndrome, inflammatory bowel diseases (IBD), iron overload.

- HSCT-comorbidity index (CI) and the European society for Blood and Marrow Transplantation (EBMT) risk score.

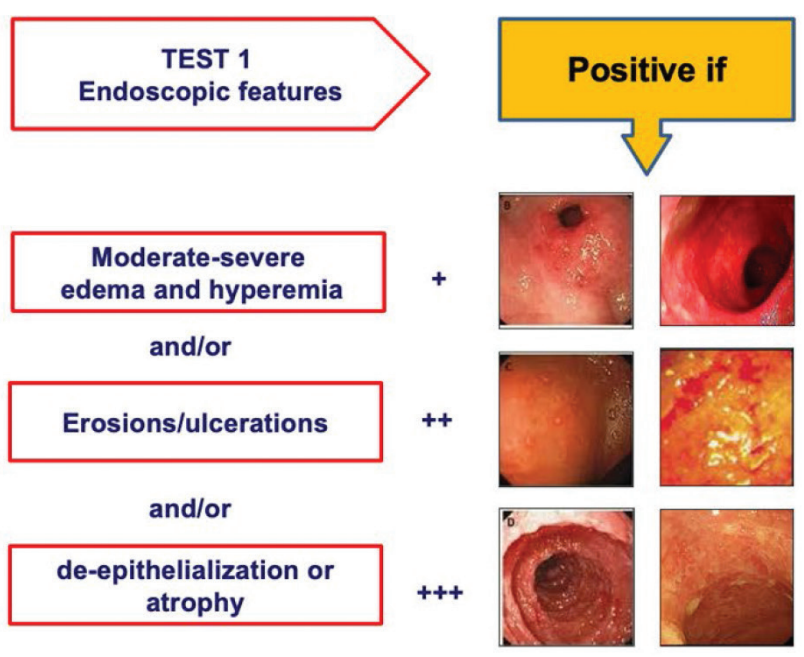

- Hematological disease and disease status before allo-HSCT.

- Conditioning regimen categorized according to EBMT criteria.

- Donors' features, including human leukocyte antigen (HLA) compatibility and stem cell (SC) source [bone marrow (BM), peripheral blood (PBSC), cord blood (CSC)].

- GVHD prophylaxis.

Patients were monitored for GI symptoms, from the day of transplant up to day 100. An esophago-gastro-duodenoscopy (EGDS) was performed in all patients with persistent ( $>6$ days) upper digestive tract symptoms. A rectal-sigmoid-colonoscopy (RSCS) was performed in case of lower GI symptoms, such as persistent diarrhea with or without hematochezia.

Endoscopic tests were considered "positive" and eventually suspicious of acute GVHD when moderate/severe oedema and hyperaemia and/or erosions/ulcerations and/or areas of disepitelization or atrophy were detected (Figure 1). Random biopsies on apparently healthy mucosa or lesion-guided biopsies were performed during every endoscopic procedure, and histological evaluation of paraffin-embedded tissue sections was performed by an experienced pathologist. The diagnostic assessment of GVHD was conducted in accordance with Shulman criteria [11]. Standard tissue staining or more sophisticated immune staining were set up, to research pathogen antigens or cytoplasmatic inclusions.

Stool cultures for bacterial, parasite and virus infections were carried out to exclude Clostridium difficile (CD), Campylobacter, Yersinia, Pathogen E. Coli, CMV, adenovirus, norovirus, herpes viruses, Giardia and Cryptosporidium. Biochemical tests that explored liver, pancreatic and intestinal functions were performed twice weekly.

Based on the results of the above-mentioned examinations, the clinical diagnosis was categorized as follows:

- Mucositis of superior digestive tract, mainly the most severe forms, classified with grade 3-4, according to the WHO criteria.

- Mucositis of inferior digestive tract with the same criteria above mentioned.

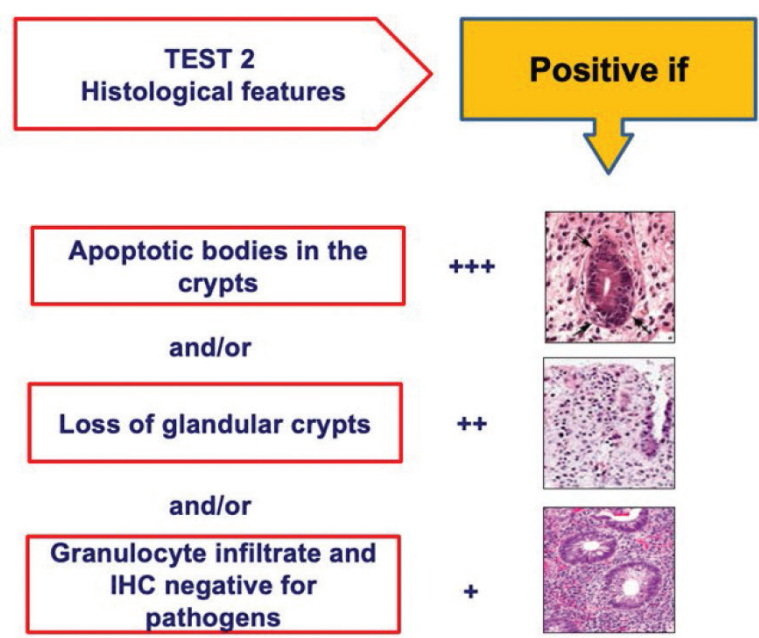

Figure 1 Endoscopic and histological features considered suspicious, suggestive or compatible with the diagnosis of GVHD. 
- Infectious complications: bacterial, viral, or parasitic agents, detected by specific cultures or toxin detection and, seldom, through endoscopic/histological procedures.

- Vascular complications such as digestive hemorrhages and ischemic events.

- Acute GVHD (aGVHD): "upper" for gastroduodenal localization; "lower" for intestinal localization and "mixed or diffuse" type when generalized in the whole digestive tract. aGVHD was classified according to the MAGIC criteria [12].

\subsection{Ethics}

This study was managed according to the principles of good clinical practice as established on the Helsinki declaration.

\section{RESULTS}

\subsection{Patient Characteristics}

The median age at transplant was 58 years with a prevalence of male gender $(57.3 \%)$. Patients underwent transplant for different hematological diseases: acute myeloid leukemia (AML) in 63 patients (51.6\%), lymphomas in 17 (13.9\%), myeloproliferative neoplasms (MPNs) in 16 (13.1\%), acute lymphoid leukemia (ALL) in $12(9.8 \%)$, myelodysplastic syndromes (MDS) in six (4.9\%), multiple myeloma (MM) in four (3.3\%), aplastic anemia (AA) in two $(1.6 \%)$ and chronic lymphatic leukemia (CLL) in two patients (1.6\%). The donor was a matched sibling in 34 patients $(28 \%)$, matched or mismatched unrelated volunteer in 54 (44\%), while 34 patients received transplant from a haploidentical donor. SC source was PBSC in 69 patients (56.5\%), BM in 48 (39.4\%), and PBSC combined with $\mathrm{BM}$ in five $(4 \%)$ patients. The conditioning regimen was myeloablative (MAC) in 85 patients $(69.6 \%)$ and reduced intensity (RIC) in 28 (23\%); 10 patients received a TBI-including regimen. GVHD prophylaxis varied according to the kind of donor source, as shown in Table 1.

\subsection{Gastro-intestinal Complications (GICs)}

Ninety-four patients (77\%) experienced GICs following alloHSCT (Table 2). Different (overlapping) kinds of GICs were often reported in the same patient. Mucositis was the most frequent complication, reported in 79 patients (65\% of overall patients and $84 \%$ of the GICs). An infective diarrhea was diagnosed in eight patients, three cases were sustained by CD; three cases were due to $\mathrm{CMV}$, and two were associated to common bacterial pathogens (Escherichia coli and Enterococcus faecium). GVHD was diagnosed in 35 patients ( $29 \%$ of all patients; $37 \%$ of those with GICs). Among thse, 20 patients presented a localized gastroduodenal GVHD while 15 developed a generalized gut GVHD involvement. A concomitant liver involvement due to GVHD was diagnosed in five of them, while skin involvement was detectable in 25 patients. As shown in detail in Table 2, all patients with GI-GVHD had a clinical grade $\geq 2$. Therapeutic management of patients with GVHD, is shown in Table 3 and distinguished as 1 st and 2nd lines when the latter was necessary.
Table 1 Characteristics of the whole cohort of 122 patients who underwent allo-HSCT

\begin{tabular}{|c|c|}
\hline & $(n=122)$ \\
\hline Age at transplant, median (range) & $58(20-71)$ \\
\hline Males, $n(\%)$ & $70(57.3)$ \\
\hline \multicolumn{2}{|l|}{ Haematological disease (\%) } \\
\hline AML & $63(51.6)$ \\
\hline ALL & $12(9.8)$ \\
\hline Lymphomas & $17(13.9)$ \\
\hline MDS & $6(4.9)$ \\
\hline MPNs & $16(13.1)$ \\
\hline MM & $4(3.3)$ \\
\hline SAA & $2(1.6)$ \\
\hline CLL & $2(1.6)$ \\
\hline \multicolumn{2}{|l|}{ Disease status, $n(\%)$} \\
\hline Remission (CR1, CR2, MRD \pm ) & $72(59)$ \\
\hline Other than remission (PR1, PR2, SD) & $50(41)$ \\
\hline Comorbidity & \\
\hline HCT-CI risk score, $n(\%) \geq 3$ & $44(36.1)$ \\
\hline \multicolumn{2}{|l|}{ Donor's compatibility, $n(\%)$} \\
\hline Sibling donor & $34(28)$ \\
\hline Haploidentical donor & $34(28)$ \\
\hline Unrelated donor $\geq 8 / 8$ & $40(33)$ \\
\hline Unrelated donor $<8 / 8$ & $14(11)$ \\
\hline \multicolumn{2}{|l|}{ Haemopoietic stem cells source, $n(\%)$} \\
\hline PBSC & $69(56.5)$ \\
\hline $\mathrm{BM}$ & $48(39.4)$ \\
\hline $\mathrm{PBSC}+\mathrm{BM}$ & $5(4)$ \\
\hline Major AB0 incompatibility, $n(\%)$ & $21(17.2)$ \\
\hline \multicolumn{2}{|l|}{ Conditioning regimen, $n(\%)$} \\
\hline MAC & $85(69.6)$ \\
\hline $\mathrm{MAC}+\mathrm{TBI}$ & $10(8.2)$ \\
\hline RIC & $28(23)$ \\
\hline nMAC & $9(7.4)$ \\
\hline \multicolumn{2}{|l|}{ GVHD prophylaxis, $n(\%)$} \\
\hline CsA/FK506 + MTX & $76(62.3)$ \\
\hline CsA/FK506 + ptCy & $45(36.7)$ \\
\hline $\mathrm{CsA}+\mathrm{MMF}$ & $1(0.8)$ \\
\hline ATG & $69(56)$ \\
\hline
\end{tabular}

AML, acute myeloid leukemia; ALL acute lymphoid leukemia;

MDS, myelodysplastic syndrome; MPNs, myeloproliferative neoplasms; MM, multiple myeloma; SAA, severe aplastic anemia; CLL, chronic lymphatic leukemia; $\mathrm{CR}$, complete remission; $\mathrm{MRD}$, minimal residual disease; $\mathrm{PR}$, partial remission; PBSC, peripheral blood stem cell; BM, bone marrow; MAC, myeloablative conditioning; RIC, reduced-intensity conditioning; nMAC, non-myeloablative conditioning; GVHD, graft-versus-host disease; CsA, cyclosporine A; MTX, methotrexate; ptCy, post-transplant cyclophosphamide; MMF, mycophenolate mofetil; ATG, antithymocyte globulin; TBI, total body irradiation.

\subsection{Diagnostic Reliability of Endoscopic and Histological Procedures to Discriminate GVHD from other Transplant Complications}

Thirty-four patients with a suspicion of GI-GVHD were evaluated by endoscopy. Nine of them, presenting with both upper and lower symptoms, underwent EGDS + RSCS plus biopsies; 26 patients with predominantly upper symptoms were investigated exclusively with EGDS, while 17 patients with mainly lower symptoms underwent RSCS only. We compared data emerged from endoscopic and histological procedures with "a posteriori diagnosis" of GVHD based 
Table 2 Gastro-intestinal complications after allo-HSCT $(n=94)$

\begin{tabular}{|c|c|}
\hline $\begin{array}{l}\text { Gastro-intestinal (GI) and hepatic } \\
\text { complications, } n=94(77 \%)\end{array}$ & $n$ (\%overall; \%GICs) \\
\hline Upper mucositis & $79(65 ; 84)$ \\
\hline Lower mucositis & $45(37 ; 48)$ \\
\hline Diffuse mucositis & $36(30 ; 38)$ \\
\hline Digestive hemorrhages & $7(5.7 ; 7.4)$ \\
\hline Infective diarrhea $n(\%)$ & $8(6.5 ; 8.5)$ \\
\hline Clostridium difficile & 3 \\
\hline $\mathrm{CMV}$ & 3 \\
\hline Escherichia coli & 1 \\
\hline Enterococcus faecium & 1 \\
\hline GI-aGVHD, $n(\%)$ & $35(29 ; 37 \%)$ \\
\hline Gastro-duodenal & 20 \\
\hline Intestinal (diffuse) & 15 \\
\hline aGVHD staging for GI symptoms & $n$ \\
\hline Stage 1 & 16 \\
\hline Stage 2 & 11 \\
\hline Stage 3 & 3 \\
\hline Stage 4 & 5 \\
\hline GI-aGVHD with liver involvement & $5(4 ; 5.3)$ \\
\hline Upper GI Stage 1 & 4 \\
\hline Lower GI Stage 1 & 0 \\
\hline Lower GI Stage $2(+1$ upper GI) & 1 \\
\hline Lower GI Stage 3 (+ 1 upper GI) & 1 \\
\hline Lower GI Stage 4 (+ 2 upper GI) & 3 \\
\hline Liver Stage 1 & 2 \\
\hline Liver Stage 2 & 1 \\
\hline Liver Stage 3 & 2 \\
\hline Liver Stage 4 & 0 \\
\hline GI-aGVHD with skin involvement & $25(20.5 ; 26.6)$ \\
\hline Upper GI Stage 1 & 22 \\
\hline Lower GI Stage 1 (+3 upper GI) & 3 \\
\hline Lower GI Stage 2 (+5 upper GI) & 7 \\
\hline Lower GI Stage 3 (+2 upper GI) & 3 \\
\hline Lower GI Stage 4 (+2 upper GI) & 2 \\
\hline Skin Stage 1 & 3 \\
\hline Skin Stage 2 & 19 \\
\hline Skin Stage 3 & 3 \\
\hline Skin Stage 4 & 0 \\
\hline GI-aGVHD with skin and liver involvement & $3(2.4 ; 3.2)$ \\
\hline $\begin{array}{l}\text { aGVHD overall clinical grade (only in GI } \\
\text { aGVHD patients) }\end{array}$ & $n$ \\
\hline Grade 1 & 0 \\
\hline Grade 2 & 23 \\
\hline Grade 3 & 6 \\
\hline Grade 4 & 6 \\
\hline \multicolumn{2}{|l|}{ aGVHD overall clinical grade (all patients) } \\
\hline Grade 1 & 31 \\
\hline Grade 2 & 25 \\
\hline Grade 3 & 12 \\
\hline Grade 4 & 7 \\
\hline
\end{tabular}

CMV, cytomegalovirus; GI-aGVHD, gastro-intestinal-acute GVHD; GVHD, graftversus-host disease; HSCT, hematopoietic stem cell transplant.

on the comprehensive analysis according to MAGIC criteria taking into account the cutaneous and hepatic localizations of the disease and an eventual post-mortem diagnostic ascertainment. With this approach, we retrospectively re-evaluated all patients' characteristics, particularly those for whom an apoptotic cryptitis was not found in the intestinal biopsies, even though other lesions such as a loss of glandular crypts or a granulocyte infiltrate were detected,
Table 3 Treatment strategies for the patients affected by acute GVHD

\begin{tabular}{lcc}
\hline & $\begin{array}{c}\text { Overall } \\
\text { patients, } \boldsymbol{n}\end{array}$ & $\begin{array}{c}\text { GI-GVHD } \\
\text { patients, } \boldsymbol{n}\end{array}$ \\
\hline Treatment 1st line & 64 & 35 \\
Patients treated & 30 & 11 \\
Topic & 16 & 9 \\
Steroid_low dose & 8 & 7 \\
Steroid_high dose & 7 & 6 \\
ECP + Steroid & 1 & 1 \\
ECP + Steroid + Vedolizumab & 2 & 1 \\
Rituximab & $n$ & $n$ \\
Treatment 2nd line & 25 & 19 \\
Patients treated & 7 & 5 \\
Topic & 4 & 2 \\
Steroid high dose & 1 & 1 \\
ECP + Topic & 7 & 5 \\
ECP + Steroid & 1 & 1 \\
ECP + Rituximab & 1 & 1 \\
ECP + Vedolizumab & 1 & 1 \\
ECP + Steroid + Vedolizumab + Etanercept & 1 & 1 \\
ECP + Steroid + Vedolizumab + Tocilizumab & 2 & 2 \\
Ruxolitinib &
\end{tabular}

ECP, extracorporeal photopheresis; GI, gastro-intestinal; GVHD, graft-versus-host disease.

with a probable or possible but not certain diagnosis of GVHD [11]. In total, 24 patients underwent both (upper and lower) endoscopic procedures, with a definitive diagnosis of GI-GVHD.

Figure 2 shows that among patients investigated with EGDS, 18 of 26 had an ascertained diagnosis of GVHD. Upper endoscopy resulted indicative of a possible GVHD in 21 patients with an absolute sensitivity (100\%) but a poor specificity (30\%); thus, this test showed a bad concordance with the final diagnosis. Histological assessment showed a probable or compatible picture for GVHD in 10 patients. The sensitivity was $60 \%$ and specificity $70 \%$ while the concordance with definitive diagnosis, (calculated with Coen's $K$ ), was 0.42 . Regarding lower GI tract evaluation, endoscopy showed mucosal lesions suggestive of GVHD in 11 of 17 patients of whom 14 received after a confirmed diagnosis of GVHD. Thus, the sensitivity and specificity of lower endoscopy were 80 and 100\%, respectively, and its concordance with the diagnosis was 0.56. Finally, histological evaluation on rectal biopsies demonstrated a sensitivity of $80 \%$ and specificity of $100 \%$ [positive predictive value (PPV) $100 \%$, negative predictive value (NPV) 60\%] and a good concordance with the final diagnosis (concordance of 0.68) (Table 4). Overall, it appears that there is a clear role of biopsy procedures, performed even on healthy mucosa. Moreover, rectal biopsy proved to be the most reliable test (Table 5).

\section{DISCUSSION}

Allo-HSCT has the potential to cure malignant (e.g., leukemias, lymphomas) and non-malignant disorders (e.g., hemoglobinopathies), but it is associated with relevant GI toxicity, particularly in the early period post-transplantation. Several conditions emerged as risk factors capable of influencing morbidity and mortality, among which are the conditioning regimen (e.g., chemotherapy, myeloablative therapy, radiation), status of the underlying cancer and severity of GVHD [1,2]. 


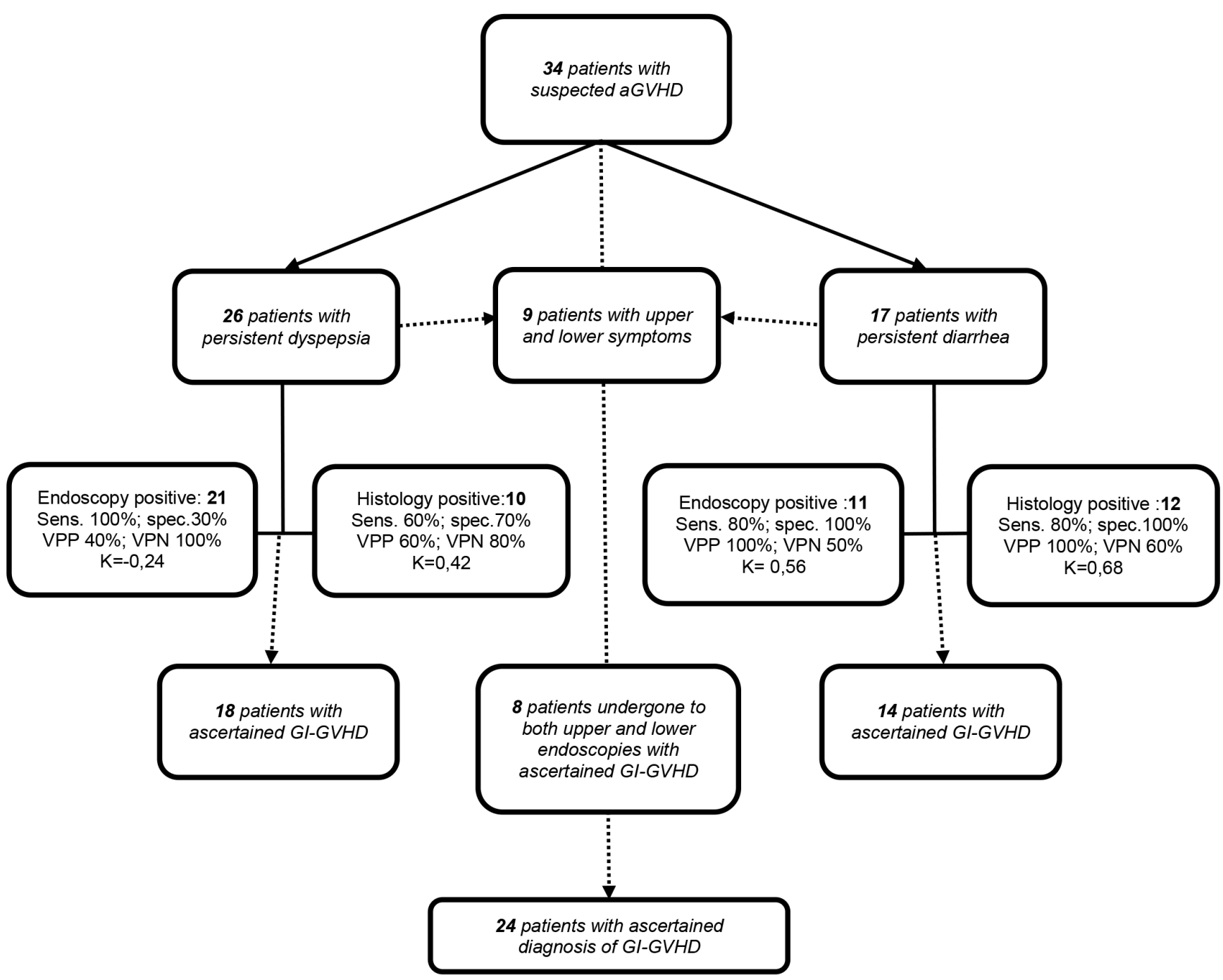

Figure 2 Flow-chart of patients undergoing the endoscopic/histological procedures upon suspicion of GI-GVHD. PPV, positive predictive value; NPV, negative predictive value; $K$ of Cohen for concordance; GI-GVHD, gastro-intestinal-graft versus host disease.

Table 4 Reliability measures of endoscopic and histological investigations for the diagnosis of acute gastro-intestinal-graft versus host disease (GI-GVHD)

\begin{tabular}{|c|c|c|c|c|c|c|}
\hline & Sensitivity & Specificity & PPV & NPV & $K$ & Concordance \\
\hline Esophagus-gastro-duodenoscopy & $1.0(0.8-1.0)$ & $0.3(0.1-0.5)$ & $0.4(0.2-0.6)$ & $1.0(0.8-1.0)$ & 0.24 & Low \\
\hline "Upper" histology & $0.6(0.4-0.8)$ & $0.7(0.6-0.9)$ & $0.6(0.4-0.7)$ & $0.8(0.6-0.9)$ & 0.42 & Moderate \\
\hline Rectum-sigmoidoscopy & $0.8(0.5-0.9)$ & $1.0(0.7-1.0)$ & $1.0(0.8-1.0)$ & $0.5(0.3-0.7)$ & 0.56 & Moderate \\
\hline "Intestinal" histology & $0.8(0.6-0.9)$ & $1.0(0.8-1.0)$ & $1.0(0.8-1.0)$ & $0.6(0.3-0.8)$ & 0.68 & Good \\
\hline
\end{tabular}

$(95 \% \mathrm{CI}) ; K$ di Cohen: $<0.01$ = null; $0.01-0.20=$ poor; $0.21-0.40=$ minor; $0.41-0.60=$ moderate; $0.61-0.80=$ high; $0.81-1.00=$ excellent. PPV, positive predictive value; $\mathrm{NPV}$, negative predictive value.

It has been estimated that GICs can substantially affect the prognosis of allo-HSCT in terms of morbidity and 100-day post-HSCT mortality $[1,2,13,14]$.

In our experience, GICs occurred in a large percentage of patients (77\%); almost all these patients developed mucositis and subsequent liver impairment. The mucositis, a clear expression of drug's toxicity, could play a role in the pathogenesis of acute liver impairment too. In fact, in a multivariate analysis, a diffuse mucositis emerged as a variable independently associated to veno-occlusive disease (VOD) ( $p=0.01$; OR 6.7; 95\% CI 1.52-29.7) in our cohort of patients. Mucositis deeply affects morbidity and early nonrelapse-mortality (NRM); it can also affect the quality of life and prolong the hospitalization with a significant economic impact [15]. The MAC regimen represents a recognized risk factor for mucositis. In fact, severe mucositis (WHO grades 3-4) occurred in approximately two-thirds of patients who underwent myeloablative conditioning for HCT [16]. 


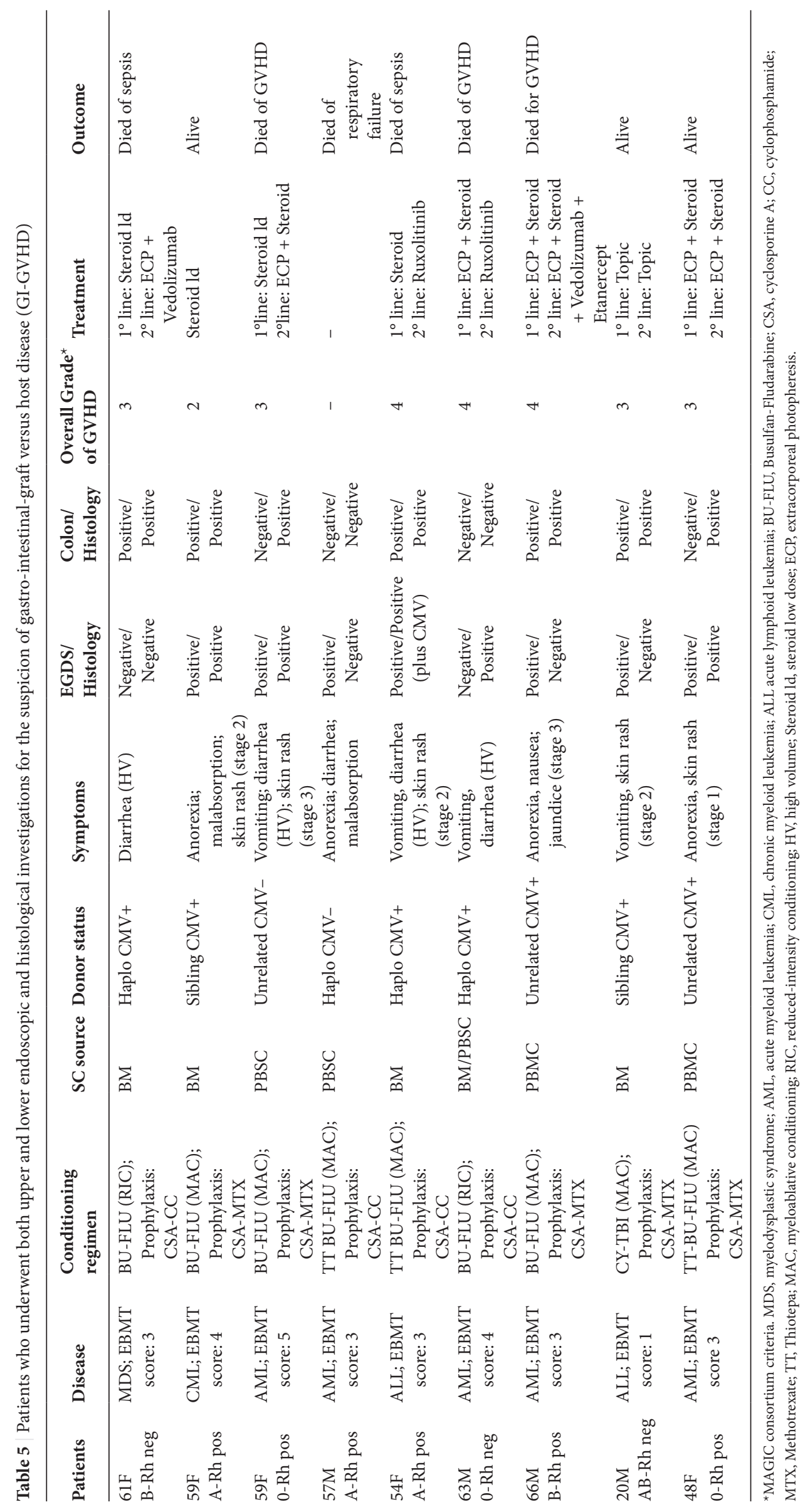


Acute GVHD is a common complication of allo-HSCT. The GI tract is involved in many of the patients who usually present with diarrhea and abdominal pain, but may also manifest nausea, vomiting, and anorexia. In our experience, aGI-GVHD occurred in $29 \%$ of patients after an allo-HSCT with a grade $>2$ in around $16 \%$ of the cases, and an associated mortality rate of about $29 \%$ of these cases. The confirmation of GVHD diagnosis often requires pathologic evaluation of tissue obtained by endoscopic biopsies. Opportunistic infections of the GI tract, principally those due to CMV, may mimic an acute GI-GVHD or they can overlap and bring out a diagnosis of GI-GVHD, hence the importance of selective microbiological staining for pathogens on the biopsy specimen.

Few studies investigating the role of endoscopic/histological evaluations in the ascertainment of GI-GVHD, have been reported. All agree on the conclusion that many of the cases of acute GI-GVHD can be identified by rectal biopsy. However, a negative rectal biopsy does not rule out the diagnosis, as the negative predictive value was not absolute [17-20].

In our population, the commonly recognized histological markers of GVHD were the cryptitis with apoptotic bodies and/or loss of glandular crypts and/or granulocyte infiltrate without an immunohistochemistry positive for pathogens [11,21].

The performance of the endoscopic-driven pathological evaluation in our population was in line with the above-mentioned studies. In our experience, rectal biopsy showed higher sensitivity and specificity than a biopsy of the upper GI tract. However, considering specifically the patients who underwent both investigations (upper and lower endoscopies), a biopsy collection guided by symptoms allowed a better diagnostic confidence; in fact, for patients who had a prevalent upper symptom, the gastric and duodenal biopsies had a NPV higher than $80 \%$. Thus, we would recommend starting with upper or lower endoscopy according to symptoms and eventually switch to the other evaluation if no mucosal lesions are detected.

Specialists from many disciplines should be involved in the management of the patient who undergoes HSCT, mainly in the allogeneic setting; moreover, a close coordination between the different disciplines is mandatory, particularly in the challenging setting of GICs. In our center, we have set up a multidisciplinary "haemato-gastro" team with a shared standardized path, aimed to quickly support the diagnostic suspicion of GVHD and to accelerate the start of treatment. This approach achieved a significant improvement in diagnosis and in the timely management of patients with GICs after allo-HSCT. We recognize that our experience is limited to a little cohort of patients so far, and it is not empowered for statistical analysis at this point. However, the first results of the present study reporting an example of a gastroenterology teamwork dedicated to patients undergoing allo-HSCT are encouraging. In our vision, a multidisciplinary approach including an aggressive GI assessment with endoscopic and histological evaluation could allow a prompt GVHD diagnosis and treatment, which might improve patient outcome. Furthermore, GI complications following allo-HSCT represent a constellation of clinical entities including GVHD, endothelial damage, and infections. A thorough GI assessment might help in distinguishing such diverse complications, thus leading to a tailored treatment.

\section{CONFLICTS OF INTEREST}

The authors declare they have no conflicts of interest.

\section{AUTHORS' CONTRIBUTION}

GT, MP, GM, IS and AO contributed to the design of the study. GT, MP, FS, LM and GG contributed to the implementation of the research. GT, MP and AF analyzed the data. GT, MP, FS and AO wrote the manuscript. $\mathrm{AO}, \mathrm{LM}$ and $\mathrm{AB}$ reviewed the manuscript. GT, MP, GM, IS, AO, MVD, FM, LD, MM and EB were involved in clinical management of the patients.

\section{DISCLOSURES}

No conflict of interest for any of the authors. The authors did not receive any financial support. All the authors designed the study, contributed patients, helped to collect patient information, performed statistical analysis, and wrote the paper.

\section{REFERENCES}

[1] Schulenburg A, Turetschek K, Wrba F, Vogelsang H, Greinix HT, Keil F, et al. Early and late gastrointestinal complications after myeloablative and nonmyeloablative allogeneic stem cell transplantation. Ann Hematol 2004;83;101-6.

[2] Barker CC, Anderson RA, Sauve RS, Butzner JD. GI complications in pediatric patients post-BMT. Bone Marrow Transplant 2005;36;51-8

[3] Spencer GD, Hackman RC, McDonald GB, Amos DE, Cunningham BA, Meyers JD, et al. A prospective study of unexplained nausea and vomiting after marrow transplantation. Transplantation 1986;42;602-7.

[4] Cox GJ, Matsui SM, Lo RS, Hinds M, Bowden RA, Hackman RC et al. Etiology and outcome of diarrhea after marrow transplantation: a prospective study. Gastroenterology 1994;107;1398-407.

[5] Peterson DE, Bensadoun RJ, Roila F. Management of oral and gastrointestinal mucositis: ESMO clinical practice guidelines. Ann Oncol 2011;22;vi78-vi84.

[6] Vera-Llonch M, Oster G, Ford CM, Lu J, Sonis S. Oral mucositis and outcomes of allogeneic hematopoietic stem-cell transplantation in patients with hematologic malignancies. Support Care Cancer 2007;15;491-6.

[7] Sonis ST, Oster G, Fuchs H, Bellm L, Bradford WZ, Edelsberg J, et al. Oral mucositis and the clinical and economic outcomes of hematopoietic stem-cell transplantation. J Clin Oncol 2001;19;2201-5.

[8] Labrador J, López-Corral L, Vazquez L, Sánchez-Guijo F, Guerrero C, Sánchez-Barba M, et al. Incidence and risk factors for life-threatening bleeding after allogeneic stem cell transplant. Br J Haematol 2015;169;719-725.

[9] Robak K, Zambonelli J, Bilinski J, Basak GW. Diarrhea after allogeneic stem cell transplantation: beyond graft-versus-host disease. Eur J Gastroenterol Hepatol 2017;29;495-502.

[10] Liakopoulou E, Mutton K, Carrington D, Robinson S, Steward CG, Goulden NJ, et al. Rotavirus as a significant cause of 
prolonged diarrhoeal illness and morbidity following allogeneic bone marrow transplantation. Bone Marrow Transplant 2005; 36;691-4.

[11] Shulman HM, Kleiner D, Lee SJ, Morton T, Pavletic SZ, Farmer E, et al. Histopathologic diagnosis of chronic graft-versus-host disease: National Institutes of Health Consensus Development Project on Criteria for Clinical Trials in Chronic Graft-versusHost Disease: II. Pathology Working Group Report Biol Blood Marrow Transplant 2006;12;31-47.

[12] Harris AC, Young R, Devine S, Hogan WJ, Ayuk F, Bunworasate $\mathrm{U}$, et al. International, multicenter standardization of acute graftversus-host disease clinical data collection: a report from the Mount Sinai Acute GVHD International Consortium. Biol Blood Marrow Transplant 2016;22;4-10.

[13] Iqbal N, Salzman D, Lazenby AJ, Wilcox CM. Diagnosis of gastrointestinal graft-versus-host disease. Am J Gastroenterol 2000;95;3034-8.

[14] Zeiser R, Socie G, Blazar BR. Pathogenesis of acute graft-versushost disease: from intestinal microbiota alterations to donor $\mathrm{T}$ cell activation. Br J haematol 2016;175;191-207.

[15] Jones JA, Qazilbash MH, Shih YT, Cantor SB, Cooksley CD, Elting LS. In-hospital complications of autologous hematopoietic stem cell transplantation for lymphoid malignancies: clinical and economic outcomes from the Nationwide Inpatient Sample. Cancer 2008;112;1096-105.
[16] Wardley AM, Jayson GC, Swindell R, Morgenstern GR, Chang J, Bloor R, et al. Prospective evaluation of oral mucositis in patients receiving myeloablative conditioning regimens and haemopoietic progenitor rescue. Br J Haematol 2000;110;292-9.

[17] Aslanian H, Chander B, Robert M, Cooper D, Proctor D, Seropian $\mathrm{S}$, et al. Prospective evaluation of acute graft-versus-host disease. Dig Dis Sci 2012;57;720-5.

[18] Ratanatharathorn V, Nash RA, Przepiorka D, Devine SM, Klein JL, Weisdorf D, et al. Phase III study comparing methotrexate and tacrolimus (prograf, FK506) with methotrexate and cyclosporine for graft-versus-host disease prophylaxis after HLA-identical sibling bone marrow transplantation. Blood 1998;92;2303-14.

[19] Ross WA, Ghosh S, Dekovich AA, Liu S, Ayers GD, Cleary $\mathrm{KR}$, et al. Endoscopic biopsy diagnosis of acute gastrointestinal graft-versus-host disease: rectosigmoid biopsies are more sensitive than upper gastrointestinal biopsies. Am J Gastroenterol 2008;103;982-9.

[20] Sultan M, Ramprasad J, Jensen MK, Margolis D, Werlin S. Endoscopic diagnosis of pediatric acute gastrointestinal graftversus-host disease J Pediatr Gastroenterol Nutr 2012;55; 417-20.

[21] Woodruff JM, Hansen JA, Good RA, Santos GW, Slavin RE. The pathology of the graft-versus-host reaction (GVHR) in adults receiving bone marrow transplants. Transplant Proc 1976;8; 675-84 